\title{
An evaluation of nasal bone and aperture shape among three South African populations*
}

Jennifer L. McDowell, MSc ${ }^{1}$

Michael W. Kenyhercz MS ${ }^{2}$

Ericka N. L'Abbé PhD, D-ABFA ${ }^{1}$

${ }^{1}$ Department of Anatomy, University of Pretoria, Private Bag x 323, Arcadia, 0007, South Africa

${ }^{2}$ Department of Anthropology, University of Alaska, Fairbanks, AK, 99775

Corresponding author:

Ericka N. L’Abbé, PhD, D-ABFA

Department of Anatomy

University of Pretoria

Private Bag x 323

Arcadia 0007

South Africa

Email: ericka.labbe@up.ac.za

Tel: +27 12 319-2438

Fax +27 $12319-2240$

\section{Highlights}

-Examined nasal aperture shapes among three socially defined South African groups.

-Three statistical methods were used to assess within and between group variation.

-Variation in nasal shape was noted among all groups and between sexes.

-Early colonialism, slavery and modern segregation shaped skeletal variation.

-No extreme trait expression as described in forensic pathology and anthropology texts.

\footnotetext{
* This research was funded by an NRF-CSUR grant (South Africa): EN L'Abbé, SD Ousley and the NRF incentive funding for unrated researchers (South Africa)
} 


\begin{abstract}
Reliable and valid population specific standards are necessary to accurately develop a biological profile, which includes an estimation of peer-reported social identification [1]. During the last 300 years, colonialism, slavery and apartheid created geographic, physical and social divisions of population groups in South Africa. The purpose of this study was to evaluate variation in nasal bone and aperture shape in a modern population of black, white, and coloured South Africans using standard craniometric variables and geometric morphometrics, namely general Procrustes and elliptical Fourier analyses.
\end{abstract}

Fourteen standard landmarks were digitally recorded or computationally derived from 310 crania using a 3D coordinate digitizer for discriminant function, principal components and generalized Procrustes analyses. For elliptical Fourier analysis, outlines of the nasal aperture were generated from standardized photographs. All classification accuracies were better than chance; the lowest accuracies were for coloured and the highest accuracies were for white South Africans. Most difficulties arose in distinguishing coloured and black South African groups from each other. Generally, misclassifications were noted between the sexes within each group rather than among groups, which suggests that sex has less influence on nasal bone and aperture shape than ancestry. Quantifiable variation in shape of the nasal aperture region between white and non-white South African groups was observed.

Keywords: ancestry, geometric morphometrics, ellipitical fourier analysis, generalized Procrustes analysis, craniometry.

Estimation of peer-reported social identity is important to the development of a biological profile from unknown skeletal remains [1]. However, medico-legal practitioners are often unfamiliar with the range of human variation associated with ancestry such that forensic pathology texts often describe the classification of crania into three broad groups, namely Negroid, Caucasoid, and Mongoloid [2]. The traditionally broad typological approach to evaluating ancestry defines nasal aperture width as long and narrow in 'Caucasoid' or 'European' groups; wide and round in 'Negroid' or 'African' groups; and intermediate among 'Mongoloid' or 'Asian' groups [2-4]. The typological approach used to describe skeletal features does not consider the complex variation within biology and thereby presents an inaccurate method for the assessment of unknown skeletal remains $[1,5]$.

Within the last 300 years, colonialism, slavery and apartheid created divisions of social groups in South Africa. From 1913 to 1983 in South Africa, approximately 60 segregation Acts concerning: race, employment, education, land tenure and geography, marriage, political representation, and state 
security were implemented to control and to govern the apartheid regime (6,7). In 1927, the first Act for a prohibition on inter-racial marriage was passed and later again in 1949 and 1950. The early 1950's saw the most devastating implementations of apartheid with the Population Registration Act No. 30, which empowered the 1951 census bureau to racially classify every South African into four groups: "Native/Bantu" (Black); Coloured, White, and Asian. The social designation of coloured refers to a highly admixed group of people, primarily from the Western Cape, that are comprised of influences from indigenous Khoikhoi and San, Asian, European, and slaves from various African and non-African countries $[9,10]$. For the purpose of this article, the terms black, coloured and white refer specifically to South African populations.

Once classified, the Group Areas Act of 1950 determined where a person could live. Black South Africans were assigned to one of thirteen designated homelands outside the Republic of South Africa. Within the Republic, whites, coloured and Indian groups were separated. The geographical separation of socially defined groups reduced the likelihood of gene flow among them. For the purpose of redress, the former apartheid classification system remains in use today; the three largest socially identified population groups are black (80\%), coloured (9\%), and white (8\%) [11].

The concept of race, specifically social race, or ancestry, is a highly debated topic within the field of anthropology. While race is not a valid biological concept, it is a sociological reality. However, even though race is not real, the social consequences of race have resulted in biological manifestations, likely through positive assortative mating [25]. While South Africa has had a storied history of forced segregation through a variety of aforementioned legislation that limited rates of intermarriage, sociologists have long identified the proclivity for people to marry within their respective social groups. Kalmijn [26] identified three causes for the persistence of endogamy: (1) a preference for spouses who are phenotypically similar; (2) other members of the same social group (friends, family, etc.) act as third party interference; and (3) limited access to members of different social groups. Each of the three potential causes for endogamy have been demonstrated legally through the various legislative acts described above, and have persisted socially as is shown through the census, in which people have self-identified as black, coloured, or white [11]. Yet, Omi and Winant [27] demonstrated that racial identification is also a sociopolitical consequence that is constantly in flux. Within the realm of population structures, anthropologists are tasked with constructing biological profiles to narrow down a potential missing persons list. Therefore creation and utilization of statistically valid methodologies are necessary to inform the anthropologist as to the probable social identity of an individual in life. In order to do this, researchers need to search for the biological manifestations of social race, which are the products of positive assortative mating.

Previous studies demonstrated osteometric and morphological differences in the crania and postcrania of black and white South Africans [5,12-15]. Yet, only one study [5] has visually assessed non-metric 
trait distribution among white, black and coloured South Africans. The results of L'Abbé et al. [5] demonstrated relatively distinct morphological differences in ancestry and sexual dimorphism for midfacial features (particularly nasal apertures width and inter-orbital breadth) among all groups. The purpose of this study was to use standard linear measurements and geometric morphometrics (GM), namely general Procrustes analysis (GPA) and ellipitical Fourier analysis (EFA) to further elucidate sexual dimorphism and social identity in mid-face shape and form among black, coloured and white South Africans. Principal component analysis (PCA) and discriminant function analysis (DFA) were used to examine the variation both within and between groups.

\section{Materials and Methods}

A total of 310 crania of black, coloured and white South Africans were digitized for this study (Table 1). The crania were collected from the Pretoria Bone Collection (University of Pretoria); the Raymond A. Dart Collection (University of the Witwatersrand); and the Kirsten Collection (Stellenbosch University).

Table 1 : Demographics and sample size (n) of South African crania digitized for study

\begin{tabular}{lccc}
\hline & \multicolumn{3}{c}{ Sex } \\
Population group & Male $(M)$ & Female $(F)$ & Total \\
\hline Black (B) & 99 & 91 & 190 \\
Coloured(C) & 23 & 13 & 36 \\
White (W) & 42 & 42 & 84 \\
Total & 164 & 145 & 310 \\
\hline
\end{tabular}

The Pretoria Bone and Raymond A. Dart Collections are cadaver-based and contain $19^{\text {th }}$ and $20^{\text {th }}$ century skeletons of known age, sex and population group - the majority of which are black and white [16,17]. In contrast, the Kirsten collection, which is also cadaver-based and of known age and sex, primarily contains coloureds.

Exclusion criteria included: (1) persons younger than 18 years of age due to the potential effects of continued growth and development on the mid-face; (2) macroscopic pathology, ante- and postmortem fractures, as these do not represent normal variation; and (3) extensive ante-mortem tooth loss (less than 6 teeth remaining), as tooth loss and subsequent alveolar resorption may change facial shape [18].

Fourteen standard landmarks of the mid-face were digitized with a 3D coordinate digitizer (Table 2). The accuracy of the 3D coordinate digitizer was 0.015 -inches $(0.38 \mathrm{~mm})$. The computer program 3Skull [19] was used to collect the raw coordinate data as well as to calculate standard craniometric measures from the coordinate data. Additionally, 5 standard craniometric variables were derived from 
Table 2 : Definition of landmarks collected from the midface

\begin{tabular}{|c|c|c|}
\hline Assigned \# & Landmark & Definition \\
\hline 1 & Alare R & Most lateral point on right side of nasal aperture \\
\hline 2 & Alare L & Most lateral point on left side of nasal aperture \\
\hline 3 & Dacryon $\mathrm{R}$ & $\begin{array}{l}\text { Point where lacrimal, frontal and maxillary bones meet on } \\
\text { medial wall of right orbit }\end{array}$ \\
\hline 4 & Dacryon L & $\begin{array}{l}\text { Point where lacrimal, frontal and maxillary bones meet on } \\
\text { medial wall of left orbit }\end{array}$ \\
\hline 5 & Glabella & $\begin{array}{l}\text { Most forward projecting point of frontal bone in the } \\
\text { midline of the supraorbital ridges }\end{array}$ \\
\hline 6 & Nasion & Midline junction of the nasal and frontal sutures \\
\hline 7 & Nasale inferius $\mathrm{R}$ & Most inferior point on right nasomaxilla suture \\
\hline 8 & Nasale inferius L & Most inferior point on left nasomaxilla suture \\
\hline 9 & Nasale superius $\mathrm{R}$ & Point at junction of right nasomaxilla and frontal sutures \\
\hline 10 & Nasale superius L & Point at junction of left nasomaxilla and frontal sutures \\
\hline 11 & Naso-dacryal subtense point & The deepest point on the nasal bone profile \\
\hline 12 & Most inferior nasal boarder $\mathrm{R}$ & Most inferior point on right border of nasal aperture \\
\hline 13 & Most inferior nasal boarder L & Most inferior point on left border of nasal aperture \\
\hline 14 & Subspinale & $\begin{array}{l}\text { Most posterior midline point on premaxilla between the } \\
\text { anterior nasal spine and prosthion }\end{array}$ \\
\hline
\end{tabular}

8 of the landmarks, which includes : inter-orbital breadth (DKB), nasal breadth (NLB), nasal height (NLH), nasal-dacryon angle (NDA), and nasal-dacryon subtense point (NDS).

For the EFA, photographs were taken of each specimen in the Frankfurt Horizontal plane using a craniophore. The midpoint of the camera was focused on the nasion of each cranium. The same camera was used to take each photograph at a distance of $46 \mathrm{~cm}$ from the tripod shaft to the back legs of the craniophore. Outlines of the nasal aperture were traced in Adobe Photoshop CS4 and formatted for entry into Shape 1.3 for EFA [20].

\section{Statistical Analysis}

As a means to examine overall shape differences as opposed to single measurements or numerical descriptors, the standard craniometric variables (SCV), Procrustes coordinates, and elliptical Fourier descriptors (EFD) are subjected to PCA. Principal Components Analysis compresses and transforms correlated variables into a series of uncorrelated composite variables, known as principal components (PC). Principal components are loaded by each of the variables in the analysis and the contribution of each variable can be examined by the relative weight, either positive or negative. The first principal component (PC1) accounts for more of the observed variance than subsequent PCs within the sample and primarily reflects overall size; however, due to the isometric scaling inherent in GPA and EFA, PC1 reflects the greatest trend in overall shape. Principal component loadings are used to assess possible landmarks permutations in metrical analyses and the contribution of each landmark to nasal aperture shape variation within the sample in the morphometric analyses [15]. Effective principal components were extracted from the appropriate data matrix to be used in subsequent analyses. . 
In order to address shape analyses independent of size, GPA was conducted on the raw 3D coordinate data in MorphoJ [21]. Raw coordinates were subjected to a Procrustes fit, or superimposition, and converted to Procrustes shape coordinates. The mean centroid of the entire sample was used to scale the landmark's raw coordinates and to rotate the entire sample to minimize the squared distances between landmarks. The Procrustes shape coordinates were subjected to the aforementioned PCA, based on the variance-covariance matrix, and shape changes could then be visualized as principal components of shape instead of merely abstract $\mathrm{x}, \mathrm{y}$, or $\mathrm{z}$ coordinates. The resulting graphs, known as lollipop graphs show the negative extreme of the PC scores at the lollipop and the stem shows, incrementally, the positive change in PC units. In sum, the lollipop graphs show the direction and magnitude of shape change wherein the longer the stem, the more marked the change.

Elliptical Fourier analysis is a method that describes the outlines of closed contours through the use of best-fit ellipses known as harmonics. The number of harmonics necessary to define a shape adequately varies depending on the complexity of the shape. The inclusion of each additional harmonic will, in turn, better define the outline shape. However, the minimum number of harmonics should be used to maximize the shape of the fit without introducing extraneous noise [20].

Each specimen's outline was entered into Shape 1.3 [20] and was aligned on its long axis in anatomical position. Once each specimen's outline was entered into the program and rotated, they were scaled to match the size of the first harmonic applied to the first specimen in the dataset. Thus, data were scaled and not strictly size-free, although gross size was removed. A mean shape was generated from the entire sample, independent of biological parameters, based on EFD.

Data for SCV and GM were analyzed in Fordisc 3.1 (FD3.1) [22] custom database using DFA to classify individuals into one of the six reference groups (BM, BF, WM, WF, CM and CF). Like PCA, DFA compresses and transforms variables into discriminant functions, or canonical variates, that best maximize between group variation instead of within group variation as with PCA. All results were cross-validated using leave-one-out-cross-validation (LOOCV) [22]. In LOOCV, upward bias towards greater classification accuracy is avoided as the individual being evaluated is removed from the reference group and tested against all other members of that group. The parameters are recalculated and the individual is then classified into one of the reference groups. Additionally, forward Wilks stepwise selection was used on each model to select only those variables that contributed to the group separation.

Fordisc 3.1 [22] was also used to calculate the Mahalanobis' generalized distances $\left(\mathrm{D}^{2}\right)$ for each specimen from its group mean as well as to examine biological distance, and significance, between groups. 


\section{Results}

Principal components analysis

Principal component analysis of the SCV resulted in five PC (Table 3). PC1 accounted for 53.9\% of the variation, while PC2, PC3, PC4 and PC5 accounted for 25.9\%, 12.2\%, 7.2\% and 0.1\%, respectively. Black and coloured females demonstrated comparable mean PC1 scores, while coloured males demonstrated slight intermediacy between blacks and whites (Fig. 1). Coloured females presented the greatest variability.

Table 3 : Principal component loadings from the standard craniometric variables (SCV).

\begin{tabular}{llllll}
\hline & PC1 & PC2 & PC3 & PC4 & PC5 \\
\hline DKB & -0.418 & -0.521 & -0.299 & 0.574 & 0.366 \\
NDA & $\mathbf{- 0 . 5 8 5}$ & 0.099 & 0.241 & 0.252 & $\mathbf{- 0 . 7 2 5}$ \\
NDS & 0.466 & -0.445 & -0.493 & 0.050 & -0.583 \\
NLB & -0.429 & -0.472 & -0.055 & $\mathbf{- 0 . 7 6 8}$ & -0.003 \\
NLH & 0.285 & $\mathbf{- 0 . 5 4 5}$ & $\mathbf{0 . 7 7 9}$ & 0.121 & -0.004 \\
\hline
\end{tabular}

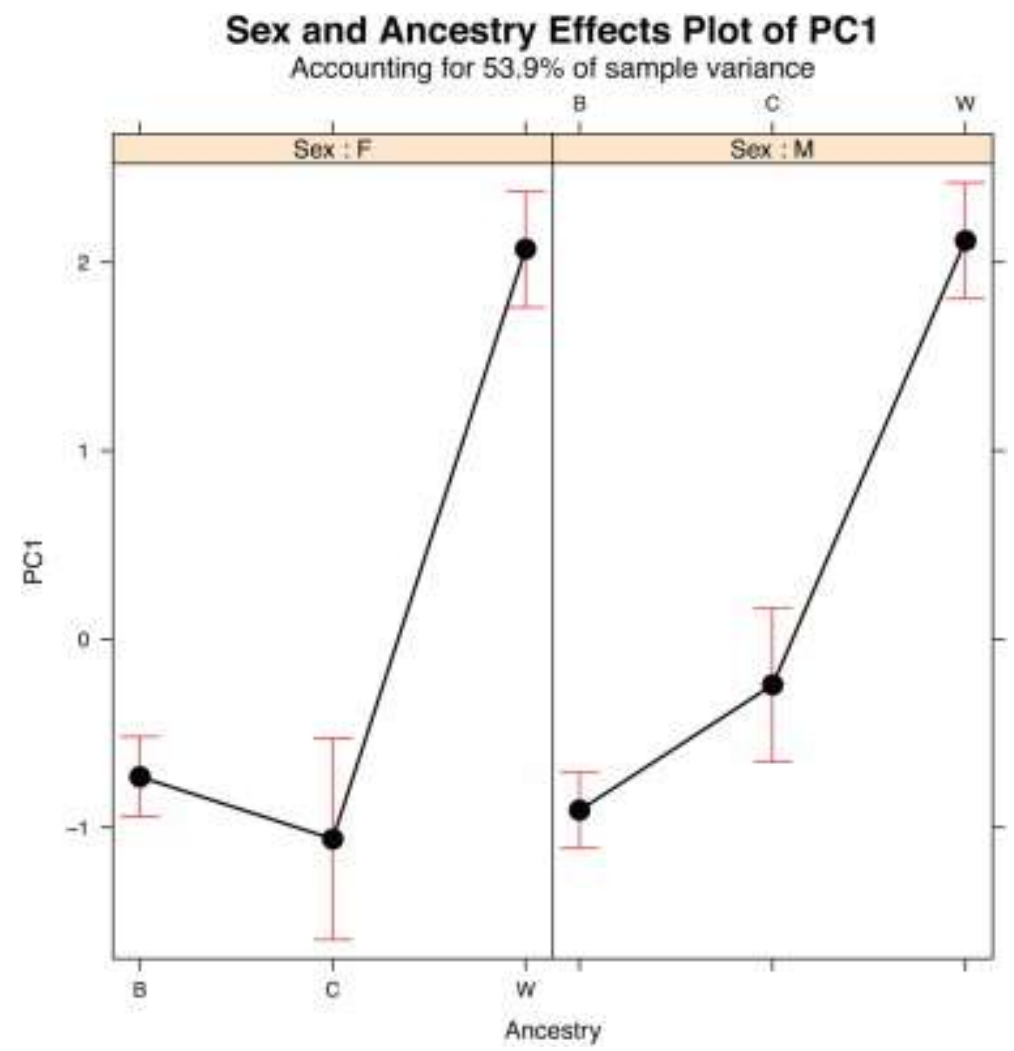

Fig. 1 : Sex and ancestry effects plot with $95 \%$ confidence intervals for PC1 of SCV. 
The PCA of the Procrustes coordinates yielded 35 PCs. The first 20 PC contributed eigenvalues greater than the eigenvalue average and comprised a total of $92.8 \%$ of the entire variation. The first PC contributed $17.3 \%$ of the variation and was most heavily influenced by the superomedial movement of nasale inferius and the inferomedial movement of alare (Fig. 2). In Figure 2, a lollipop graph was generated from MorphoJ [21] in which the lollipop circle represents the negative extreme of the shape and the stem represents, incrementally, the change in shape associated with an addition of one PC score unit. The longer the stem, the more marked the shape differences. Black and coloured South Africans demonstrated comparably positive PC1 means (represented by the stems), while whites presented a negative PC1 mean (represented by the lollipop). Whites demonstrated a lower nasale inferius when compared to both black and coloured groups. Similar to the SVCs, black and coloured females demonstrated PC1 values in contrast to the white females, while coloured males demonstrated a slight intermediacy to whites, while showing a greater affinity towards black males (Fig. 2).

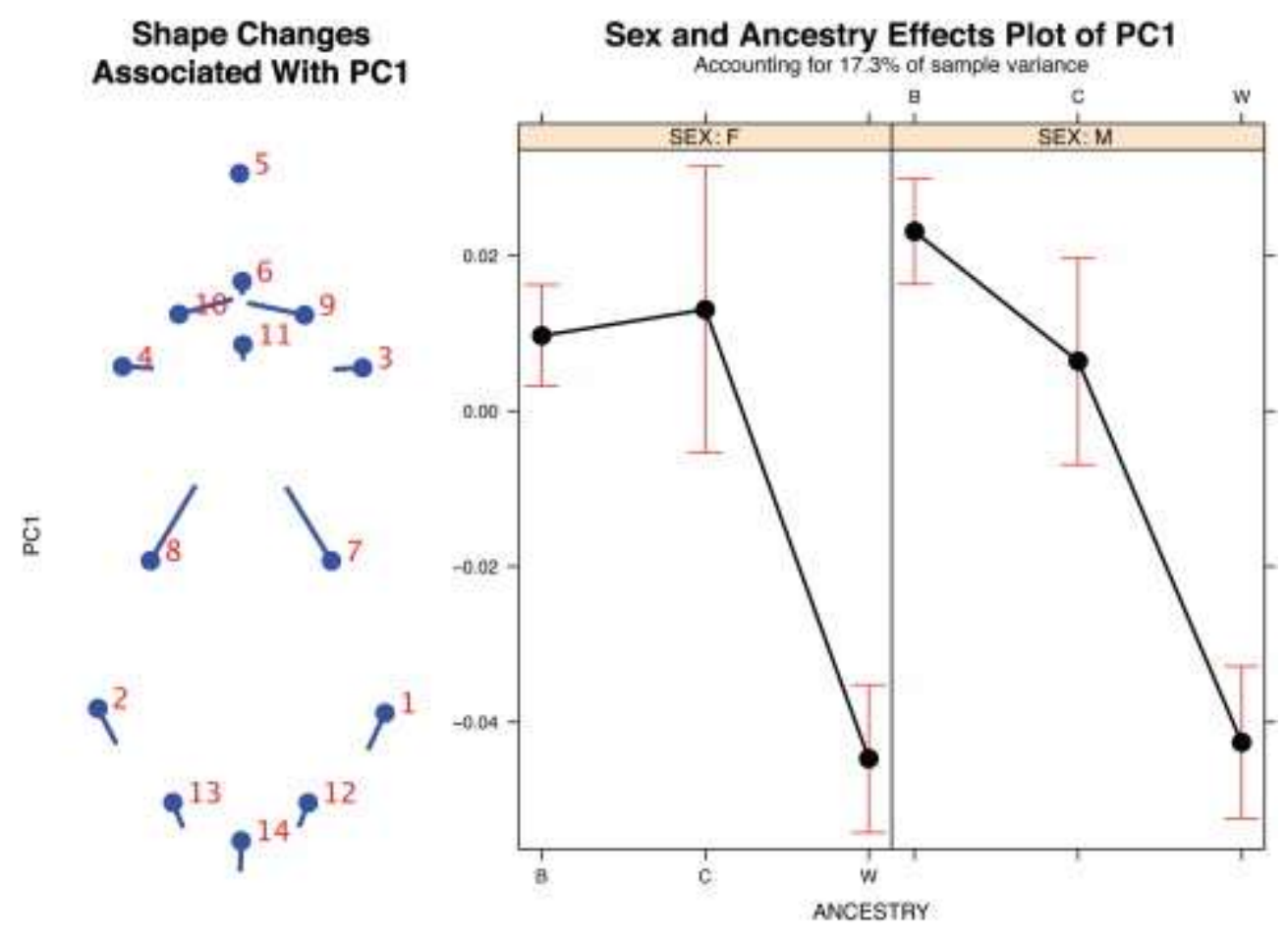

Fig. 2 : Shape changes associated with Procrustes coordinates in PC1 and sex and ancestry effects plot with 95\% confidence interval. Whites demonstrate the negative extreme (lollipop circle), while blacks and coloureds demonstrate the positive extreme (lollipop stems).

The PCA of the EFD yielded 40 PC with the first 10 PCs contributing eigenvalues greater than $1 / \mathrm{n}$ [20]. Principal component 1 accounted for $41.3 \%$ of the sample variation and demonstrated that black South Africans (males and females) present with shorter and rounder nasal aperture shapes with slightly superior extension on the inferior nasal border than either coloured or whites (Fig. 3). Coloured females have nasal aperture outlines more similar to black females, while coloured males 
present intermediate nasal aperture outlines between black and whites. White South Africans (males and females) have a pear-shaped nasal aperture outline (Fig. 3).

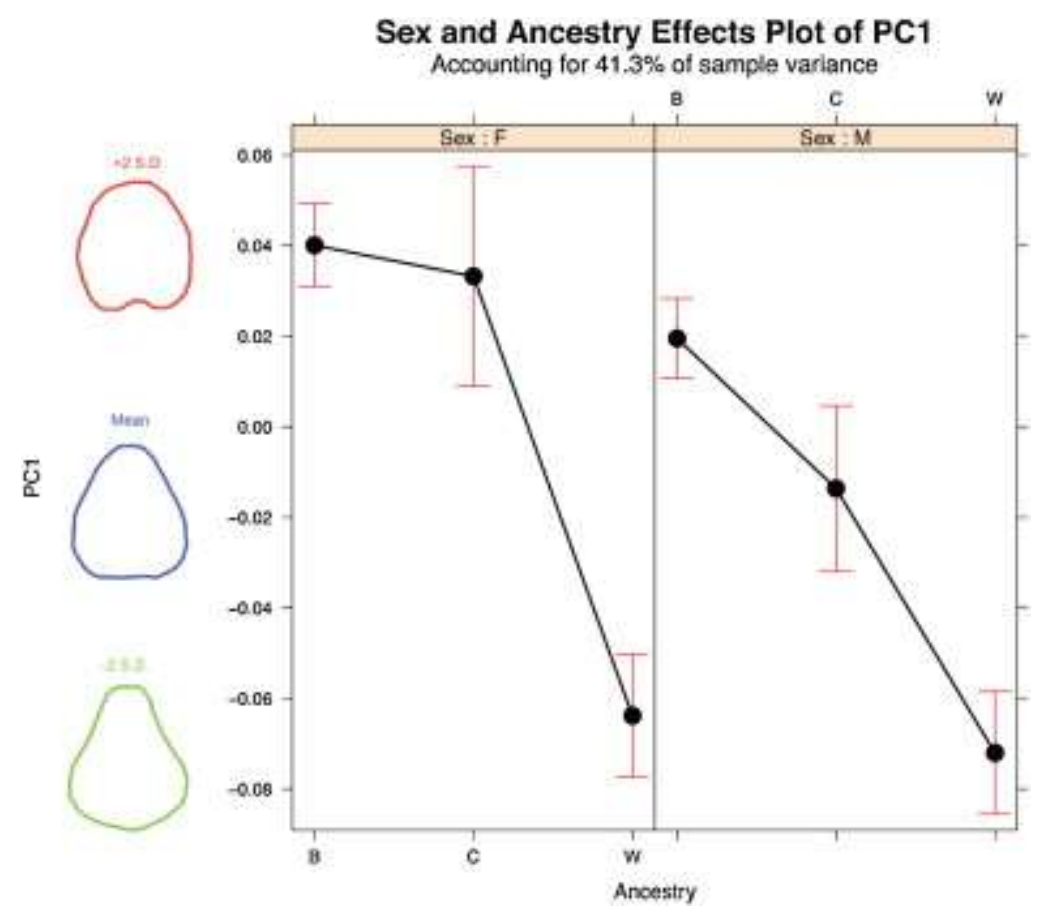

Fig. 3 : Sex and ancestry effects plot on the right and corresponding shape changes as determined through EFA on the left.

\section{Discriminant Function Analysis}

Six-way Sex and Ancestry

Total correct classifications ranged from 42.3-59.7\% (Table 4). The SCV achieved the greatest total correct classifications while the EFA yielded the lowest.

Table 4 : Total correct classifications for each sex and ancestry group and each method (SCV, GPA, EFA)

\begin{tabular}{|c|c|c|c|c|c|c|c|}
\hline \multirow[b]{2}{*}{ Analysis } & \multicolumn{6}{|c|}{ Percent Correct (\%) } & \multirow{2}{*}{$\begin{array}{l}\text { Total Correct } \\
(\%)\end{array}$} \\
\hline & $\mathrm{BF}$ & BM & $\mathrm{CF}$ & $\mathrm{CM}$ & WF & WM & \\
\hline SCV & 38.2 & 54.5 & 46.2 & 41.7 & 71.4 & 65.9 & 52.3 \\
\hline GPA & 51.0 & 60.9 & 30.8 & 48.0 & 81.4 & 70.7 & 59.7 \\
\hline EFA & 48.4 & 32.3 & 30.8 & 26.1 & 50 & 57.1 & 42.3 \\
\hline
\end{tabular}

The six-way DFA of sex and ancestry using the five SCV (NDA, NLH, DKB, NDS, and NLB) resulted in a total correct classification of $52.3 \%$ (Table 4 and Fig. 4). All groups' $\mathrm{D}^{2}$ were significantly different $(\mathrm{p}<0.05)$ except for coloured males and black females $(\mathrm{p}=0.201)$ as well as coloured females and black females $(p=0.052)$. The PC of Procrustes coordinates resulted in a total correct classification of $59.7 \%$, using five forward Wilks stepwise selected variables: PC1, PC2, PC3, 
PC4, PC5, and PC12 (Table 4). All groups' $\mathrm{D}^{2}$ were significantly different $(\mathrm{p}<0.001)$, except for coloured females and black females $(p=0.552)$ and coloured females and black males $(p=0.055)$. Using the PC of EFA with the forward Wilks stepwise selected variables: PC1, PC2, PC5, PC6, and PC9 resulted in a total correct classification of $51.8 \%$ (Table 4). All groups' $\mathrm{D}^{2}$ were significantly different $(\mathrm{p}<0.05)$.

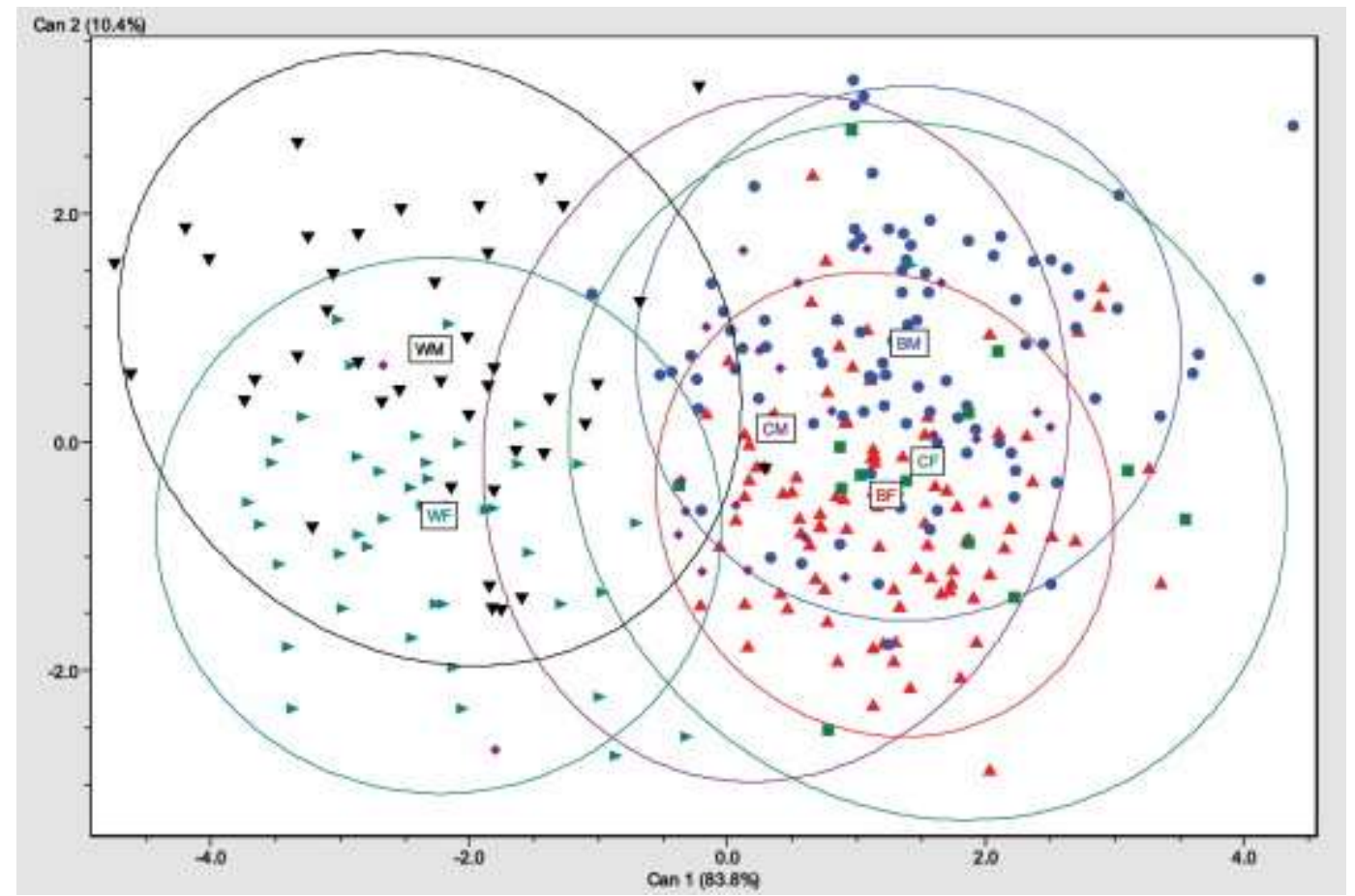

Fig. 4 : A canonical variate scatterplot of the PCs of the Procrustes coordinates accounting for $94.2 \%$ of the variation, showing the results of the six-way sex and ancestry discriminant function analysis. Both white males and white females are distinct from the other population groups. The same trends were observed for each of the six-way analyses (SCV and EFA).

Black females classified between $38.2 \%$ and $51 \%$ correctly and black males classified between $32.3 \%$ and $60.9 \%$ correctly for all methods (Table 4). Black females misclassified most frequently as black males, whereas black males misclassified most frequently as coloured males/females. Coloured females classified $30.8 \%$ to $46.2 \%$ correctly and coloured males classified between $26.1 \%$ and $48 \%$ correctly (Table 4). Coloured females classified most frequently as black female and classified second most commonly as themselves, except in SCV when it was the other way around. With all three methods coloured males classified most commonly as themselves and misclassified most frequently as black female. White females classified between $50 \%$ and $81.4 \%$ correctly and white males classified between $57.1 \%$ and $70.7 \%$ correctly (Table 4 ). White females and males misclassified most frequently as each other using all three methods. 
Three-way Ancestry

Total correct classifications ranged from 65.2-76.8\% correct classifications (Table 5). The PCs of the Procrustes coordinates achieved the greatest total correct classifications and the EFA yielded the lowest total correct classifications.

Table 5 : Total correct classifications for each ancestry and method

\begin{tabular}{|c|c|c|c|c|}
\hline \multirow[b]{2}{*}{ Analysis } & \multirow[b]{2}{*}{ B } & \multicolumn{2}{|c|}{ Percent Correct (\%) } & \multirow{2}{*}{$\begin{array}{l}\text { Total } \\
\text { Correct }(\%)\end{array}$} \\
\hline & & $\mathrm{C}$ & W & \\
\hline SCV & 56.9 & 54.1 & 92.8 & 66.2 \\
\hline GPA & 72.3 & 60.5 & 94.0 & 76.8 \\
\hline EFA & 61.6 & 38.9 & 84.5 & 65.2 \\
\hline
\end{tabular}

The three-way DFA of population groups, with the sexes pooled, using five SVCs: DKB, NDA, NDS, NLB, and NLH resulted in a total correct classification of $66.2 \%$ (Table 5). All groups $\mathrm{D}^{2}$ were significantly different at $\mathrm{p}<0.001$ except for black and coloured groups $(\mathrm{p}=0.062)$. Using nine forward Wilks selected variables for the PC of Procrustes coordinates: PCs 1-3, PC5, PC7, PC12, PC13, PC15, and PC17 resulted in a 76.8\% total correct classification (Table 5 and Fig. 6). All groups $\mathrm{D}^{2}$ were significantly different at $\mathrm{p}<0.001$. When using 7 forward Wilks selected variables for the PC of EFD: PCs 1-5, PC8, and PC10 resulted in a 65.2\% total correct classification (Table 5). All groups $\mathrm{D}^{2}$ were significantly different at $\mathrm{p}<0.05$.

Blacks classified between $56.9 \%$ and $72.3 \%$ correctly and classified second most frequently as coloured and infrequently as white in all methods. Coloureds correctly classified $38.9-60.5 \%$ of the time and classified second most frequently as black and infrequently as whites in all methods; except in EFA where coloureds were just as frequently classified as black. Whites had the highest correct classification of $84.5-94.0 \%$ and, in all three methods, classified second most frequently as coloured, followed by black (Table 5 and Fig. 5). 


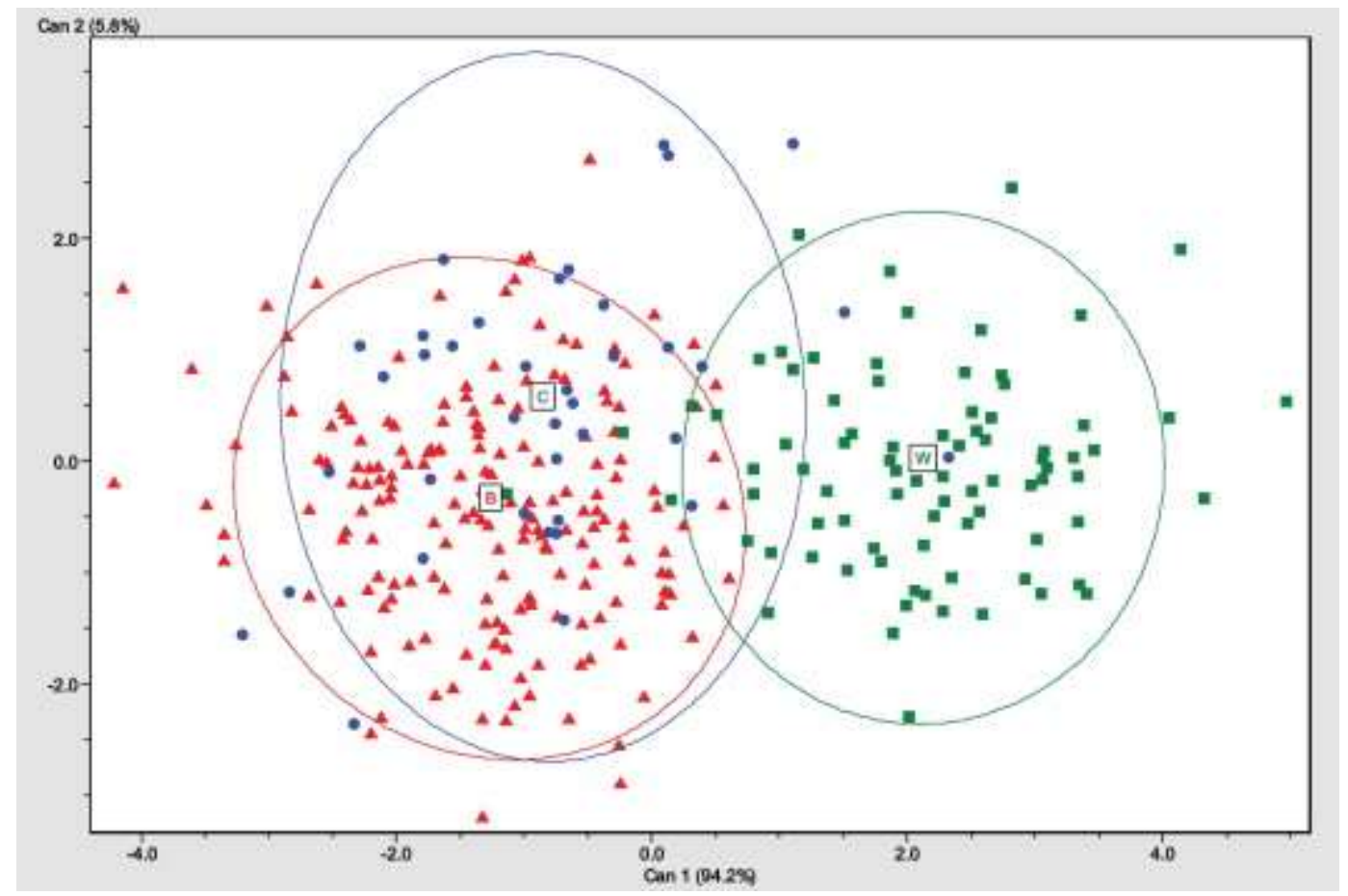

Fig. 5: A canonical variate scatterplot of the PCs of the Procrustes coordinates accounting for $100 \%$ of the variation. The trend represented in the graph was consistent for the other methods used in the three-way analyses.

\section{Discussion}

Distinct differences in mid-facial size and shape are noted among all South Africans groups and between the sexes, but none of the South African groups display extreme trait expression, as suggested in historical anthropology texts $[3,4]$.

Black South Africans often present with a wide and rounded nasal aperture, a wide inter-orbital and nasal bone width, and a short nasal height; this creates an overall larger mid-facial region in comparison to the other groups. Compared to coloureds, black groups exhibit similar inferior nasal borders but higher nasal spines that create an inverse heart-shaped nasal opening. Coloureds displayed a wider inter-orbital breadth but a relatively narrower nasal superius and are more variable in size and shape than either whites or blacks.

Whites were the most distinct of the three groups, presenting with a, sharp and acute nasal bridge (i.e. NDA), a small inter-orbital breadth, a long nasal height and a narrow nasal breadth. White South Africans also exhibited a distinct nasal aperture outline in which the superior aspect is concave with the widest portion being located inferiorly. 
Overall, the osteometric examination of size was not as robust as the quantification of shape for distinguishing variation among groups. The greater fidelity awarded by shape is further shown by the location of NDS being more informative than NDA (which is generated through NDS). The PCs of the Procrustes coordinates achieved the greatest accuracies among the groups. The relatively poorer classification accuracies associated with EFA may be attributed to the fact that only one variable, nasal aperture outlines, was considered in the analysis and likely included redundant information.

White South Africans exhibited the most sexual dimorphism of the groups; whereas Coloureds exhibited the least sexual dimorphism but the most heterogeneity among the groups. Interestingly, blacks misclassified most commonly as coloured of the same sex, but coloureds did not misclassify in the same way. While size differences contributed to sexual dimorphism within the groups, the overall shape of the mid-facial region did not change significantly within the respective groups, as demonstrated with individuals most often classifying as the correct ancestral group but the wrong sex. Despite the existence of sexual dimorphism, mid-facial morphology can be used to distinguish between ancestry groups without knowledge of the person's sex. As exhibited by the correct classifications, indeed more optimistic results were achieved without the involvement of sex. When assessing craniometric differences among the three groups, L'Abbé and colleagues [23] noted that low classification accuracies for sex and ancestry were also observed among coloured and black South Africans and may be attributed to higher heterogeneity and lower sexual dimorphism than whites.

While no discrete pattern of biological variation exists among humans, clinal morphological variation is observed in geographical distances and is often driven by social and cultural aptitudes [23-25, 28]. Population variation can be seen as a feedback loop between cultural and biological factors such that cultural views of social race create barriers to mate selection, and in turn to the distribution of human variation within a population [29].

The unique mid-facial features of whites when compared to other South African groups may be explained, but not limited to, different ancestral origins (Europe vs. Africa); early colonialism in the Western Cape; as well as forced segregation within the apartheid system. The immigration of Europeans into the Southern Cape of South Africa (late- $17^{\text {th }}$ century) and broad racial classification and forced segregation underneath the $20^{\text {th }}$ century apartheid government may partly explain differences among previously classified and now self-identified groups of people. As South Africa transforms from one society to the next, the apartheid legacy remains not in deed but in the vision of redress. Classification systems remain in use and are reverted to "when 'correcting' apparent 'misclassifications' [30]. Twenty-two years after the disbanding of apartheid legislation, social barriers continue to exist in modern South Africa [31, 32]. While no longer mandatory under the constitution, social self-classification is requested of all South Africans and is often equated to employment and educational opportunities. 
Diverse cultural/sociopolitical histories are reflected in variation among these modern social groups. White groups are distinctly different from black and coloured populations, but no clear discrimination can be made between males and females of the latter two groups, possibly due to greater similarities among themselves than to white male or female South Africans.

The accuracy of some identification methods, such as age and sexing, is ancestry dependent [35]. Therefore, it is not only important to understand and improve methods for estimating ancestry but also to understand any limitations. The problems faced with identifying coloured individuals and distinguishing between white and non-white South Africans becomes critical in providing an accurate biological profile. This study has highlighted the high variability of South African groups, particularly coloureds, and has shown how unknown individuals are likely to identify and misidentify under analysis. For medico-legal reasons it is also advantageous to be able to provide the most likely misclassifications for an unknown individual.

\section{Conclusion}

Currently, variation observed within three large populations within the country may be useful to not only estimate ancestry but to monitor future changes in the distribution of traits within a population. Large numbers of illegal immigrants; an over-load of unidentified persons within the country; and an increase in deaths makes forensic anthropology a difficult, if not always applicable, field in South Africa. Modifications to existing standards are paramount so as to bring the country and the needs of the country on par with international standards.

A statistical approach to addressing peer-reported ancestry improves our knowledge of human variation and also the manner in which we address establishing a biological profile from unidentified remains.

\section{References}

[1] Hefner J (2009) Cranial nonmetric variation and estimating ancestry. J Forensic Sci 54:985-995

[2] Saukko P, Knight B (2004) The establishment of identity of human remains. In: Knight's Forensic Pathology, $3^{\text {rd }}$ edn. Arnold, London, pp99-129

[3] Hooten EA (1947) Up from the Ape, revised edn. The Macmillan Company, New York

[4] Rhine S (1990) Non-metric skull racing. In: Gill G \& Rhine S (ed) Skeletal attribution of race: Methods for forensic anthropology, $4^{\text {th }}$ edn. University of New Mexico, Maxwell Museum of Anthropological Papers, Albuquerque, pp9-20

[5] L'Abbé EN, van Rooyen C, Nawrocki SP, Becker PJ (2011) An evaluation of cranial traits used to estimate ancestry in a South African sample Forensic Sci Int 209:1-3. doi:10.10116/j.forsciint.2011.04.002 
[6] Lemon A, (2012) Residential Segregation: apartheid. In: Smith SJ (ed) International Encyclopedia of Housing and Home. Elsevier, pp111-120

[7] Thompson L, (2001) A history of South Africa, $3^{\text {rd }}$ edn. Yale Nota Bene Press: Yale University

[8] Patterson N, Petersen DC, van der Ross RE et al (2010) Genetic structure of a unique admixed population: implications for medical research. Hum Mol Genet 19:411-419

[9] Adhikari M (20050 Contending approaches to coloured identity and the history of the coloured people of South Africa. History Compass 3:1-6

[10] Long JC, Li J, Healy ME (2009) Human DNA sequences: More variation and less race. Am J Phys Anthropol 139:23-34

[11] Statistics South Africa (2012) Census 2011: Statistical Release

[12] Franklin D, Freedman L, Milne N, Oxnard LE (2007) Geometric morphometric study of population variation in indigenous South African crania. Am J Hum Biol 19:20-33

[13] Patriquin M, Steyn M, Loth SR (2002) Metric assessment of race from the pelvis in South Africans. Froensic Sci Int 127(1-2):104-113

[14] İşcan MY, Steyn M (1999) Craniometric determination of population affinity in South Africans. Int J Legal Med 112:91-97

[15] McDowell JL, L'Abbé EN, Kenyhercz MW (2012) Nasal aperture shape evaluation between black and white South Africans. Forensic Sci Int 222(1-3): doi:10.1016/j.forsciint.2012.06.007

[16] L'Abbé EN, Loots M, Meiring JH (2005) The Pretoria bone collection: A modern South African skeletal sample. HOMO 56:197-205

[17] Dayal M, Kegley,A, Stkalj G, Bidmos M, Kuykendall K (2009) The history and composition of the Raymond A. Dart collection of human skeletons at the University of the Witwatersrand, Johannesburg, South Africa. Am J Phys Anthropol 140:324-335

[18] Reichs KM, Huber CD, Lipping WR, Tangl S (2011) Atrophy of the residual alveolar ridge following tooth loss in an historical population. Oral Dis 17:33-44

[19] Ousley SD (2004) 3Skull Version 2.1.111P. (Computer software)

[20] Iwata H, Ukai Y (2002) SHAPE: A computer program package for quantitative evaluation of biological shapes based on elliptic fourier descriptors. J Hered 93(5):384-385

[21] Klingenberg CP (2011) MorphoJ: An integrated software package for geometric morphometrics. Mol Ecol Resour 11:353-357

[22] Jantz RL, Ousley SD (2005) FORDISC 3: Computerized forensic discriminant functions. v3.1 (Computer software \& manual). University of Tennessee, Knoxville

[23] Hall M, Morris A (1983) Race and Iron Age human skeletal remains form Southern Africa: an assessment. Social Dynamics 9(2):29-36 
[24] L'Abbé EN, Kenyhercz M, Stull KE, Ousley SD (2013) Craniometric assessment of modern $20^{\text {th }}$ century black, white and "coloured" South Africans. Proceedings of the $65^{\text {th }}$ Annual Meeting of the American Academy of Forensic Sciences 19:444

[25] Ousley SD, Jantz R, Freid D (2009) Understanding race and human variation: Why forensic anthropologists are good at identifying race. Am J Phys Anthropol 139:68-76

[26] Kalmijn M. 1998. Intermarriage and homogamy: causes, patterns, trends. Annu Rev Sociol 24:395-421.

[27] Omi M, Winant H. 1994. Racial formation in the United States, $2^{\text {nd }}$ ed. New York: Routlage.

[28] Dubow S. 1995. Scientific racism in modern South Africa. Cambridge: Cambridge University Press. Pp.3.

[29] Edgar HJH, Hunley KL (2009) Race reconciled?: How biological anthropologists view human variation. Am J Phys Anthropol 139:1-4

[30] Erasmus Z (2012) Apartheid race categories: daring to question their continued use. Transformation 79:1-11 pp6

[31] Jacobson CK, Amoatong AY, Heaton TB (2004) Interracial marriages in South Africa. J Comp Fam Stud 35(3):443-458

[32] Washington Post, Kaiser Family Foundation, Harvard University (09 March 2004) South Africans at then years of democracy: public opinion and media research. http://www.kff.org/kaiserpolls/upload/Survey-of-South-Africans-at-Ten-Years-of-DemocracyToplines.pdf. Accessed 10 March 2012

[33] Oettlé A, Steyn M, (2000) Age estimation from sternal ends of ribs by phase analysis in South African blacks. J Forensic Sci 45(5):1071-1079

[34] Patriquin ML, Steyn M, Loth SR (2005) Metric analysis of sex differences in South African black and white pelves. Forensic Sci Int 147:119-127

[35] Schmitt A (2004) Age-at-death assessment using the os pubis and the auricular surface of the ilium: a test on an identified Asian sample. 14(1):1-6 